\title{
The Internal Photoelectric Effect in Crystals
}

\section{By A. H. Wruson, Cavendish Laboratory, Cambridge}

$\mathrm{T}$ HE extensive work of Gudden, Pohl and others has to a certain extent clarified the behaviour of insulating crystals which become electrical conductors under the influence of light. In these substances the photo-conductivity is entirely electronic, and we leave out of consideration electrolytic processes in which massive ions transport the current. Crystals can in the main be divided into two groups-idiochromatic crystals which are photosensitive in the pure state, and allochromatic crystals which only become photosensitive after being treated with X-rays, or which owe their sensitiveness to the presence of impurities. It has so far proved impossible to correlate these complicated photo-effects with the theory of the solid state, but in view of the recent advances in the theory of poor conductors and of the influence of impurities on conductivity, ${ }^{1}$ it seems worth while to see if any unification is now possible.

If we examine the possible types of photoeffect which can take place in what we believe to be a fairly correct model of an insulator, we find that we are able to explain a large part of the phenomenon known as the primary photoelectric current, which is that part of the current which is proportional to the intensity of the light. The explanation offered here is similar in many respects to the explanations put forward during the past decade by Gudden and Pohl, and may be considered as a justification of their main hypotheses. In what follows, the theoretical principles which govern the photo-conductivity will be outlined only, no attempt being made to discuss the subsidiary phenomena. A good account of the experimental results will be found in a recent article by $\mathrm{F}$. C. Nix. ${ }^{2}$

It is now well known that the energy spectrum of an electron moving in a perfect lattice splits up into bands of allowed and disallowed energies, and if there are just sufficient electrons present to fill up one of the allowed bands, there can be no conductivity at absolute zero temperature. Further, if an electron is placed by any process in an unoccupied band, it becomes a 'free electron', and again, if there are not quite enough electrons present to occupy a band fully, then the resulting 'holes' are free to move through the crystal, and behave like free electrons with positive charge. In an insulator in the pure state, there are just sufficient electrons present to fill up one of the bands, and any conductivity is due to the presence of impurities, or has been produced artificially by electron bombardment or by the action of light. We consider as a typical allochromatic insulator sodium chloride, and for simplicity restrict ourselves to a one-dimensional lattice of $n$ sodium atoms and $n$ chlorine atoms arranged alternately. We further idealise the problem by leaving out of account all the electrons except one of every atom, and we therefore have $2 n$ electrons to deal with. Since the sodium chloride crystal is an ionic one, the highest occupied band belongs to the chlorine atoms, and the motion of an electron in this band represents an electron jumping from one chlorine to another. This 'chlorine band' contains $n$ energy levels, equal to the number of chlorine atoms present, and these energy levels will just accommodate the $2 n$ electrons, since according to Pauli's principle not more than two electrons can ever occupy the same energy level.

When a quantum of short wave light is absorbed to sensitise the crystal, its effect is to transfer an electron from a chlorine to a sodium atom. In this state the crystal is not conducting, and so

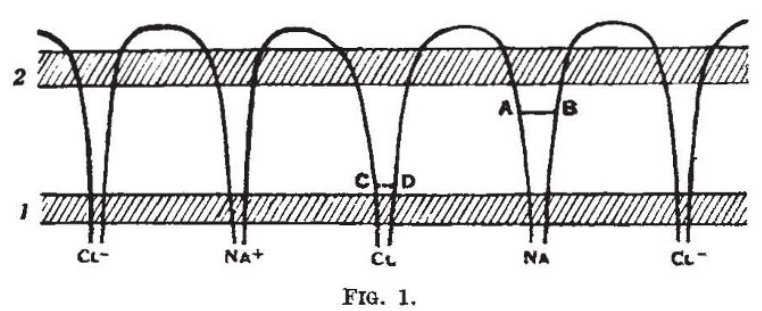

we must assume that the electron has not been excited into a band of the crystal but into a discrete energy level. The correct way of describing the crystal when it is in this sensitised state is to say that it is built up of $(n-1)$ positive sodium ions and $(n-1)$ negative chlorine ions in which are imbedded two impurities, a neutral sodium and a neutral chlorine. The energy levels of this system are illustrated in Fig. 1. The potential energy of an electron is drawn, and the shaded band 1 represents the chlorine band which is fully occupied, while the band 2 represents the lowest unoccupied band. Although the neutral chlorine and sodium are drawn as neighbours, it is not implied that this is necessarily so in an actual crystal.

The band 1 has $(n-1)$ energy levels, and these will just accommodate the $2(n-1)$ electrons belonging to the ions. The neutral sodium and chlorine have the remaining two electrons in discrete states. The energy level of the electron on the sodium is represented by $A B$, and the energy level of the 'hole' caused by the removal of the electron from the chlorine ion is represented by $C D$. The whole crystal is non-conducting provided the energy level $A B$ lies below the band 2 , and provided $C D$ lies above 1 . In thinking of these bands, it is essential to remember that they are energy bands, and not localised in space, so 
that we have drawn band 1 crossing both the sodiums and the chlorines, although it is a chlorine band. Only the discrete states can be thought of as belonging to one particular atom. The crystal in this excited state cannot be stable, but since in some cases, of which rock salt is an example, it can exist for years, it must be in a metastable state. Since any electron from the band 1 of the original crystal may be excited into a state such as $A B$, the absorption of light is continuous over a range of frequencies corresponding to the width of the band 1 . The continuous absorption is therefore due to the initial state belonging to a continuum and the final state being a discrete one, whereas in atoms and molecules it is the final state which belongs to a continuum. In rock salt the absorption which sensitises the crystal takes place in the ultra-violet and X-ray regions, though in the silver halides the absorption extends into the visible.

If the crystal is now placed in an electric field and illuminated with light of such a wavelength that the electron in the state $A B$ is ejected into the band 2, a current will be observed, and this constitutes what is known as the primary negative current. A new absorption band has therefore been introduced into the crystal at a wave-length which is usually considerably longer than that of the light required to sensitise the crystal. In rock salt this absorption takes place in the yellow. After the negative current has ceased to flow, we are left with a lattice in which there are $n$ sodium ions, $(n-1)$ chlorine ions and one neutral chlorine atom, among which are to be divided the $(2 n-1)$ electrons. Of these electrons, $2(n-1)$ are just sufficient to fill the band belonging to the chlorine ions, and the remaining one is attached to the chlorine atom in a discrete state, so that the crystal is once more non-conducting.

This is not the only possible state of the system. Another possible configuration is that in which there are $n$ positive sodium ions, and $n$ exactly similar chlorine ions with $(2 n-1)$ electrons distributed among them. In this configuration the crystal would be conducting, the conductivity being caused by the vacancy in the shell of electrons forming the chlorine band, and this 'hole' behaves like a positive electron. In rock salt this state of the crystal has greater energy than that in which one of the chlorines is neutral, and we may represent the state of affairs schematically by saying that the neutral chlorine atom has a vacant energy level $C D$, which can take up an electron from the band $l$ if an amount of energy is supplied represented by the difference in energy between $C D$ and the band 2. This energy may be supplied by illumination with infra-red light, or by heating, in which case the energy is supplied by the lattice vibrations.

Provided the temperature is low enough, it is possible to keep the crystal in the non-conducting state for a long time, and to let it pass over slowly into the conducting state, thereby separating out the positive current from the negative current, which appears immediately the crystal is illuminated. Gudden and Pohl ascribe the possibility of this separation to the sluggishness with which the 'holes' move through the crystal. According to the present theory, the 'holes' in a band can move through the lattice as quickly or nearly as quickly as the free electrons, and the sluggishness of the positive current is due to the slowness of the change from a non-conducting lattice with a neutral chlorine imbedded in negative chlorines to a conducting lattice in which all the chlorines are on the same footing.

It might quite well happen that the state $A B$ does not lie below the band 2, but actually in it. In this case $A B$ can no longer be thought of as being discrete, but belongs to the band 2 . The electron on being removed from band 1 immediately becomes a free electron, which is the characteristic of an idiochromatic crystal, a typical example being diamond. In diamond the configuration of the crystal with lowest energy, after an electron has been ejected, seems to be made up of a positively charged carbon atom imbedded in neutral carbons, and the 'hole' which is left therefore corresponds to a discrete state of exactly the same nature as $C D$ in Fig. 1. So for diamond, as for rock salt, the negative and the positive currents can be separated. In order to bring the diamond into a state in which a positive current can flow, it is necessary to supply energy in the form of infra-red light or heat, and then the crystal passes over into the state in which all the carbon atoms are on the same footing, and in which there are not quite enough electrons to fill up a band.

A further possibility is that, when an electron is ejected from the band 1 , the state of lowest energy is that in which all the atoms are on the same footing. In this case the positive and negative currents must flow simultaneously, and there would be no possibility of separating them. This case would be represented by the level $C D$ lying below the top of the band 1, a state of affairs which seems to be realised for sulphur. The 'hole' cannot then be considered as being attached to any particular atom. However, it should be borne in mind that the conducting state may merely have lower free energy than the non-conducting state, and that at very low temperatures sulphur might behave in the same way as diamond.

We may conclude from the above discussion that the theory reproduces quite naturally many of the most striking features of the internal photo-effect, and that there is no essential difference between idio- and allochromatic crystals, or between those in which the negative and positive currents can be separated and those in which they cannot. The criterion for distinguishing the various cases is whether an electron in a pure insulator goes into a discrete state such as $A B$ on absorbing light or into a band such as 2 , and whether the resulting 'hole' corresponds to a discrete state such as $C D$ or whether the 'hole' lies in the band 1 . 
It must, however, be admitted that there are still many puzzling features of the primary current. In allochromatic crystals the photoelectric response and the absorption of light correspond very well, but in idiochromatic crystals the photoelectric response has a maximum near the long wave edge of the absorption band and falls off very rapidly in the interior of the band where the absorption is large. It therefore seems that, when the absorption is large, there is no conductivity (the absorption of the light which produces the photo-effect in allochromatic crystals is always small). This seems to mean that, when many electrons are excited in say diamond, they go into discrete states and not into a band. One can see vaguely that, when many atoms have electrons excited, the original classification of states into bands, based on the properties of a perfect lattice, is likely to break down, and to be replaced by a classification based on the properties of the individual atoms, but such an explanation cannot be considered as satisfactory. This and many other problems must await further developments of the theory before they can be tackled successfully.

${ }^{1}$ A. H. Wilson, Proc. Roy. Soc., A, 138, 458; 1931 : and 134, 277 ; 1931.

2Reviews of Modern Physics, 4, 723; 1932.

\section{Hormones and Evolutior}

\section{By J. T. Cunningham}

ACCORDING to the most advanced results $A$ of genetical research, evolution is to be investigated by statistical and mathematical methods. The researches and conclusions of the geneticists are governed by the conceptions of species and divergence. It is obvious that the production of the many varieties of rabbits, dogs, and other mammals, and of birds, under domestication, to say nothing of plants, is an example of evolution; and it may possibly be to a great degree, if not entirely, explained by random mutation and survival. But the geneticists ignore many other phenomena which are equally or more important than the origin of varieties or species, especially the phenomena of adaptation. They regard survival as equivalent to selection, but that term as used by them means usually indirect selection. The survival is attributed to greater viability or fecundity, not to the utility of diagnostic characters. Genetics include extraordinary discoveries concerning sexlinkage, but almost nothing about sex-limited characters, which are known to be inherited by both sexes but developed exclusively or to a greater degree in one.

The doctrine of genes appears to be satisfactory in relation to variation and the origin of varieties and species, but it fails to explain adaptive evolution. When we consider the origin of terrestrial from aquatic vertebrates, the various adaptations of limbs to flight in birds, mammals, and some extinct reptiles, the reversed adaptation of air-breathing vertebrates to aquatic life, and many other obvious and direct adaptations, it is a truism to say that their development in the individual depends on genes in the nuclei of ova and sperms, but it has not yet been shown that they can be explained by any random mutation of the genes. The difficulty is still greater when we consider metamorphosis. It may be admitted that the metamorphosis of the flat-fish is the result of its genetic constitution, although we do not know precisely how the genes produce the hereditary characters in development. But here we have a change in important structural characters from a symmetrical phase with the median plane vertical to an asymmetrical phase with the median plane horizontal, a change taking place gradually in post-embryonic life and corresponding with the change in the relation of the fish to the direction of light and gravity.

We are asked to believe that this co-relation of structural development with external forces is not due to any effect produced by those forces, but to random mutations in the genes which were independent of the external forces, and the survival of those mutations which had the complex co-relation which we see. If we used the mathematical symbols of which the modern geneticist is so fond, how should we express the probability of the production of such an adaptation by random mutation and selection? The gene theory in this case must assume that every conceivable mutation may occur, including those which determine the change from symmetry to asymmetry in the individual, and that sooner or later each is bound to occur, so that selection is omnipotent. But there is no evidence of the occurrence under experiment or observation of the occurrence, uninfluenced by external conditions, of anything like the distortion of the skull which causes the change in the position of the eyes in the flat.fish, and leaves the symmetry of the anterior and posterior regions of the skull unaffected.

The problem of evolution can be attacked from either end of the ontogeny, from the genes to the development of the characters, or from the characters back through development to the genes. Adaptation does not consist merely in the possession of characters which are supposed to be useful or beautiful like the spots on the back of a beetle or the ocelli in a peacock's tail, but also of the functions of organs and their relation to the organism's

No. 3294, Vol. 130] 